\title{
Potassium Channel and Glioma
}

\author{
Jia Liu ${ }^{1,2}$, Chao $\mathbf{Q u}^{2,3}$, Jing-xin $\mathrm{Wu}^{2,3}$, Li-Jia $\mathrm{An}^{1}$ and Wei Zou*2,3 \\ ${ }^{1}$ School of Life Science and Biotechnology, Faculty of Chemical, Environmental and Biological Science and Technology, China \\ ${ }^{2}$ College of Life Science, China
}

${ }^{3}$ Liaoning Key Laboratories of Biotechnology and Molecular Drug Research and Development, China

*Corresponding author: Wei Zou, Liaoning Key Laboratories of Biotechnology and Molecular Drug Research and Development,

College of Life Science, Dalian 116029, China

\begin{tabular}{l}
\hline ARTICLE INFO \\
\hline Received: 幽 March 25, 2019 \\
Published: 幽 April 01, 2019 \\
Citation: Jia Liu, Chao Qu, Jing-xin Wu, \\
Li-Jia An, Wei Z. Potassium Channel and \\
Glioma. Biomed J Sci \& Tech Res 16(4)- \\
2019. BJSTR. MS.ID.002879.
\end{tabular}

Keywords: Potassium Channel; Glioma; Cell Cycle; Cell Proliferation; Cell Apoptosis
ABSTRACT

Potassium channels regulate a multitude of biological processes and play important roles in a variety of diseases by controlling potassium flow across cell membranes. They are widely expressed in the central and peripheral nervous system, and selectively conduct potassium ions across the membrane down their electrochemical gradient. As a malignant tumor derived from nerve epithelium, glioma has the characteristics of high incidence, high recurrence rate, high mortality rate, and low cure rate. Since glioma cells show invasive growth, current surgical methods cannot completely remove tumors. Adjuvant chemotherapy is still needed after surgery. Because the blood-brain barrier and other factors lead to a lower effective concentration of chemotherapeutic drugs in the tumor, the recurrence rate of residual lesions is extremely high. Therefore, new therapeutic methods are needed. Numerous studies have shown that different potassium channel subtypes are differentially expressed in glioma cells and are involved in the regulation of the cell cycle of glioma cells to arrest them at different stages of the cell cycle; also studies have shown that potassium Channels play an important role in the proliferation and apoptosis of gliomas. Increasing evidence suggests that potassium channels express in glioma cells and regulate glioma cell behaviors such as cell cycle, proliferation and apoptosis. This review article aims to summarize the current knowledge on the function of potassium channels in the different processes of glioma, suggests potassium channels participating in the development of glioma.

\section{Introduction}

Ion channels have been shown to play a pivotal role in the origin of various cancers in the literature. They become a very useful and accessible target for modulation. Among those under study, potassium channels are the best characterized with established role in cell cycle regulation. Potassium channels are transmembrane proteins that are defined by their ability to selectively facilitate the permeation of $\mathrm{K}+$ between intracellular and extracellular environments [1]. Under resting conditions, nearly all of the ions that move across the membrane (intracellular to extracellular) are $\mathrm{K}+$ ions, and these results in a negative membrane potential [1]. According to conductance properties, structural criteria, and whether combine with stimulus, potassium channels are divided into four classes: Kv channels, KCa2+channels, Kir channels and K2P channels. Potassium channels are one of the most widely distributed ion channels and play an important role in the development of many diseases [2]. Recent studies found many potassium channel subtypes abnormally expressed and regulated cell biological behaviors, such as the leiomyosarcoma aggressiveness [5,6].

Therefore, some scholars have classified the malignant tumor into the "Potassium Channel Disease" category [7-9] and regarded potassium channels as the promising therapeutic target. Accumulating evidence has proved that a variety of potassium channels, including $\mathrm{Kv}$ channel [10-12], $\mathrm{KCa}^{2+}$ channel [13], K2P channel [14] and Kir channel [15] are overexpressed in tumorous tissues compared with their healthy counterparts. Consistently, it 
was found that the potassium channels, which were significantly unusual expression in glioma, were mainly $\mathrm{Kv}$ channels (such as EAG1[16], hERG [17]), $\mathrm{KCa}^{2+}$ channels (BK [18-20], IK [18,21], KCa3.1 [22] and SK[18]) and KATP channels (such as Kir6.2 [15,23]), and they were highly correlated with the malignancy of gliomas. Potassium channel blockers such as 4-AP (4-aminopyridine, Kv blocker), tolbutamide (KATP channel blocker) could significantly influences the growth of glioma [15,24]. Therefore, potassium channels play an important role in the development of gliomas.

\section{Expressions of Potassium Channels and Gliomas}

$\mathrm{K}^{+}$channels selectively expressed in multiple types of tumor cells, and deeply influenced on biological behavior of tumors, such as proliferation, apoptosis, differentiations and invasions. However $\mathrm{K}^{+}$channels didn't expressed or down-expressed in normal tissues, such as mammary glands [24], prostate glands [25, 26]. This selective expression in tumor tissue indicates potassium channel may be the potential therapeutic target and has important clinical application value. Gliomas are the most common malignant brain tumors, and numerous studies have shown that there are many kinds of potassium channels expressed in glioma cells, such as $\mathrm{Kv}$ channels [27], BK channel [28], $\mathrm{KCa}^{2+}$ channels [29], KATP channels [30] and hERG channels [31] (Tables 1\&2). Preussat [32] first found the levels of expression of Kv1.3 and Kv1.5 subtypes discriminated between various glioma groups, and a clear differential expression of Kv1.5 was observed according to malignancy grade. Later, Debska-Vielhaber [28] found that LN229 cell (human glioma cell line) expressed BK channel. What's more, Basrai reported that BK channel was found in the human glioma cell line STrG-1 (anaplastic astrocytoma, WHO grade III) and D54-MG (glioblastic glioblastoma, WHO grade IV).

Table 1: A list of abbreviations.

\begin{tabular}{|c|c|}
\hline Abbreviation & Full name \\
\hline Kv Channel & Voltage-gated Potassium Channel \\
\hline $\mathrm{KCa}^{2+}$ Channel & Calcium-activated Potassium Channel \\
\hline Kir Channel & Inward-rectifier Potassium Channel \\
\hline $\mathrm{K}_{2 \mathrm{P}}$ Channel & Two-pore-domain Potassium Channel \\
\hline EAG Channel & Ether à go-go Channel \\
\hline hERG Channel & human Ether à go-go Related Gene Channel \\
\hline BK Channel & $\begin{array}{l}\text { Large Conductance Calcium-activated Potassium } \\
\text { Channel }\end{array}$ \\
\hline IK Channel & $\begin{array}{l}\text { Intermediate-conductance } \mathrm{Ca}^{2+} \text {-activated Potassium } \\
\text { Channel }\end{array}$ \\
\hline SK Channel & $\begin{array}{c}\text { Small-conductance } \mathrm{Ca}^{2+} \text {-activated Potassium } \\
\text { Channel }\end{array}$ \\
\hline $\mathrm{K}_{\text {ATP }}$ Channel & ATP-sensitive Potassium Channel \\
\hline
\end{tabular}

Table 2: Expression of $\mathrm{K}^{+}$channels in glioma cells.

\begin{tabular}{|c|c|c|}
\hline Channel & Tumor & Reference \\
\hline \multirow{2}{*}{ Kv Channel } & $\begin{array}{c}\text { Diffuse Astrocytoma, } \\
\text { Glioma }\end{array}$ & {$[27](\mathrm{Kv} 1.5),[32](\mathrm{Kv} 1.3$ and } \\
& & Kv1.5) \\
\hline \multirow{2}{\mathrm{KCa}^{2+}}{} & Glioblastoma & {$[29,55,56](\mathrm{KCa} 3.1),[18-20,28$,} \\
Channel & & 57] (BK), [18, 21, 58] (IK), [18] \\
& & $(\mathrm{SK})$ \\
\hline
\end{tabular}

\begin{tabular}{|c|c|c|}
\hline $\begin{array}{c}\text { Kir } \\
\text { Channel }\end{array}$ & Glioblastoma & [59] (Kir4.1), [60] (Kir4.2) \\
\hline $\begin{array}{c}\text { EAG } \\
\text { Channel }\end{array}$ & Glioblastoma & {$[16]$ (EAG1) } \\
\hline $\begin{array}{c}\text { hERG } \\
\text { Channel }\end{array}$ & Glioblastoma & {$[31]$} \\
\hline $\begin{array}{c}\mathrm{K}_{\text {ATP }} \\
\text { Channel }\end{array}$ & Glioma & {$[30,61]$} \\
\hline
\end{tabular}

EAG channel has been shown to express in numerous tumor tissues, and may closely associate with tumor generation, malignant growth, invasion and metastasis[31,33-35]. hEAG channels belong to the family of Kv channels with delayed rectifier characteristics [31]. Patt [35] found a differential expression of hEAG1 and hERG1 in gliomas depending on the malignancy grade and nature of the tumor cells. Catacuzzeno [36] demonstrated that $\mathrm{K}+$ ion flux was essential for the FCS-induced glioblastoma cell (U87-MG) migration. Not only in the human glioma cell line, but also in the animal glioma. Zhu [37] found that Kv1.3 and Kv1.6 channel could be observed in rat astrocytes. And recently, Venturini [38] report that Kv1.3 is expressed in mitochondria of human and murine GL261, A172 and LN308 glioma cells. Treatment with the novel Kv1.3 inhibitors induced massive cell death in glioma cells. These studies have supported that potassium channel may involve in the process of gliomas.

\section{Potassium Channels and Cell Cycle of Gliomas}

The cell cycle is divided into defined phases, namely G1 (first gap), S (synthesis), G2 (second gap) and M (mitosis), while a post-mitotic cell in G0 is considered to be in a non-dividing status (quiescent). While cancer cells generally maintain moderately depolarized membrane potential compared with nontransformed cells, transient hyperpolarization has been reported to be necessary for successful G1/S cell cycle progression [8,33]. In spinal cord astrocytes down-regulation of Kir accompanied with a depolarization was observed to promote cell cycle progression through the G1/S checkpoint. This indicates depolarization to be necessary for entering the S phase [39]. Using medicines blocking the Kv channels and KATP channels in U87-MG could inhibit the growth of tumor via an arrest in the G0/G1 transition during the cell cycle [30]. Samely, Huang [15] reported that treating U251(glioma cell line) cells with the blocker of KATP channels blocked cell cycle in G0/G1 phase, while a block of delayed rectifier potassium channel caused proliferating astrocytes to arrest in G0/ G1. What's more, Klumpp [40] recently reported that blocking the intermediate-conductance $\mathrm{Ca}^{2+}$-activated $\mathrm{K}+$ channel $\mathrm{KCa} 3.1$ could force G2/M cell cycle progression in GL261 glioma cells treated with the DNA-alkylating drug temozolomide, and then facilitates apoptotic cell death.

In summary, there are evidence suggesting that several different types of potassium channel-ligand-as well as voltagegated or combinations of these channels are necessary for cells to progress through the cell cycle. The reason for this effect was attributed to $\mathrm{K}+$ diffusion through potassium channels out of the 
cells as shown in theoretical models resulting in hyperpolarization of the membrane potential $[7,41]$.

\section{Potassium Channel and Proliferation/Apoptosis of Glioma}

Given the important role of potassium channels in tumors, it is necessary to clarify their role in proliferation. Accumulating evidence has indicated that potassium channels are relevant players in controlling cell proliferation and apoptosis of various tumor cells, and pharmacological blockades of $\mathrm{Kv}$ channels lead to cell proliferation inhibition [42]. Electrophysiological and pharmacological results proved that quinidine inhibited cell (U87MG cell [43] and C6 glioma cell [44]) proliferation and apoptosis in the concentration range required to block Kv channel currents. This indicates that quinidine potentially inhibited cell proliferation and induced apoptosis by blocking Kv channel activities. Since KATP was found also involved in regulating numerous cellular functions. $\mathrm{Ru}$ [30] proved that $\mathrm{Kv}$ and KATP channel blockers inhibited proliferation and tumorigenesis of U87-MG glioma cells. It was likely that potassium channels activities modulated Ca2+influx into U87-MG cells and therefore affected the proliferation and apoptosis. Huang [15] studied the effect of KATP channels activity on glioma cells proliferation, which is mediated by ERK (extracellular signalregulated kinase) activation.

They found thatactivation KATPchanneltriggered ERKactivation and inhibiting KATP channel depressed ERK activation. Abdullaev [13] proved that downregulation BK channels in U251 cells using gene-specific siRNA didn't affect the rate of proliferation, while paxilline (inhibitor of BK channel) reduced both U251 and U87-MG cells proliferation in an additive fashion. Hao [45] down-regulated TASK-1 by the transfection of siRNA improved the proliferation rates of N2A cells, suggested that this channel was involved in the regulation of neuronal growth. What's more, Staudacher [31] found that suppression of hERG protein is a crucial molecular event in glioblastoma cell (LNT229 and U87-MG) apoptosis. Recent study [43] has shown that Kv channels are expressed in the inner mitochondrial membrane. Kv3.4 inhibition blocked MPP+(1Methyl-4-phenylpyridinium ion)-induced cytochrome c release from the mitochondrial intermembrane space to the cytosol and mitochondrial membrane potential depolarization, which are characteristic features of apoptosis [46,47]. The finding of Szabo study [48] demonstrated that mitochondrial Kv1.3 channel mediated Bax-induced (Bcl-2 associated protein X) apoptosis, and Cheng [49] found that mitochondrial potassium channels play a central role in the induction of apoptosis by Bax. Similar to these, $\mathrm{Ru}$ [50] reported that blocking the $\mathrm{Kv}$ channels would induces glioma cell apoptosis by reducing expression of microRNA-10b-5p.

However, some studies hold the different opinion. DebskaVielhaber [28] showed BK channel openers CGS7181 and CGS7184 induced glioma cell death, and this effect was due to the modulation of calcium homeostasis by BK channel openers leading to activation of calpains. $\mathrm{Li}$ [51] found that the blockage of $\mathrm{Kv}$ channels could improve the proliferation of N2A cells. Potassium channels may use several mechanisms to regulate cell proliferation. The induction of tumor growth via the abnormal expression of potassium channel subtypes since $\mathrm{Ca}^{2+}$ acts as an activator involved in many cellular signal transduction pathways, including the cell growth and mitosis pathways [52]. Hyperpolarization increases the driving force for $\mathrm{Ca}^{2+}$ into the cells according to the Nernst equation, which makes sense since $\mathrm{Ca}^{2+}$ is another major factor in cell-promoting proliferation. Ca2+-sensitive potassium channels like BK channels may serve as regulatory sensors by hyperpolarizing cells and in this way limit the action of voltage-operated $\mathrm{Ca}^{2+}$ channels.

And in another hand, the cell membrane of the tumor cell is more polarized than the normal cell, and the early stage of G1 phase in cell cycle requires a brief hyperpolarization, while blocking potassium channels results in depolarization which ceases cell proliferation.The studies that whether potassium channel blockers promote tumor cells' apoptosis are rarely published. But some believe that the activation of potassium channel and the outflows of potassium and $\mathrm{Cl}$ - are necessary to change cell volume before apoptosis, thus blocking potassium channels cause the inhibition of apoptosis [41].

\section{Blockers of Potassium Channel and Treatments of Gliomas}

The multiple drug resistance (MDR) of tumor cell associated with a variety of mechanisms is a significant obstacle in tumor therapy. However, the main form of resistance related to membrane glycoprotein. Glycoproteins pump the toxic substances out of cells and make the intracellular drug concentration lower than the threshold of killing cells. In MDR phenotypic cancer cells, high expression of membrane glycoproteins is associated with increased intracellular $\mathrm{K}^{+}$current. It has been found that a large number of toxins and drugs have the ability to regulate potassium channels, which can be used to block potassium channels or change channels' sensitivities to voltage and calcium concentration. The development of the tumor requires the acceleration of proliferation and the weakening of apoptosis. The inhibition of potassium channel blockers to cell proliferation is related to cell volume, transmembrane potential, and cell cycle. Yang [53] proved blocking potassium channel using TEA (tetraethylammonium) could inhibit rat glioma cell lines (C6 and $9 \mathrm{~L}$ ) proliferation and induce apoptosis in both cell lines, and it might be associated with the increase in intracellular ROS (reactive oxygen species) production.

$\mathrm{Ru}$ [43] found that quinidine (a commonly used Kv channel blocker) significantly inhibited the proliferation of U87-MG cells and induced apoptosis in a dose-dependent manner. Sales [34] proved that silencing EAG1 is a promising strategy to improve glioma treatment. Newly, there is a compounds, senicapoc which is made with KCa3.1 blocking tool, has previously been in Phase III clinical trials. And this medicine can cross the blood brain barri- 
er, which means it would be available for repurposing, and could be used to quickly translate findings compounds into clinical trials [54]. The opening of potassium channels can release the non-voltage sensitive calcium and activate the $\mathrm{Ca}^{2+}$ channel, which makes $\mathrm{Ca}^{2+}$ enter intracellular space and participates in the $\mathrm{Ca}^{2+}$ related signaling pathway, then accelerate tumor cells' proliferation. Potassium channel blockers can inhibit this process, therefore inhibit the tumor cells' growth. Blockage of potassium channels not only can inhibit the growth of tumor cells, but also can induce the apoptosis of tumor cells. Therefore, we could view this as a potential therapeutic target in cancer treatments [55-57]. In the early stage of tumor cell generation, potassium channel blockers were used to prevent excessive proliferation, while the tumor cells were killed by potassium channel activators in the stage of terminal cancers. Therefore, inhibition of glioma cells by potassium channel blockers and its specific mechanism remains to be further studied.

\section{Summary}

The expression of $\mathrm{K}+$ channels of different subtypes has been confirmed in various glioma cells or tissues, and the blockade of $\mathrm{K}^{+}$channels often affects a variety of cellular activities. In addition, the potassium channel can also as the gene therapy target of cancer treatment. Such as using knockout, antisense oligo nucleotide can inhibit the growth of tumor. The role of $\mathrm{K}^{+}$channel inhibitors in inhibiting glioma cell growth can provide new insights into the treatment of glioma. However, the cellular mechanism regulated by $\mathrm{K}^{+}$channels is extremely extensive and the role of $\mathrm{K}^{+}$channel blockade at the level of glioma tissue is still lacking in a large amount of experimental data. Therefore, further study of the role of $\mathrm{K}^{+}$channels in the development of gliomas and verify its effects at the tissue or even the individual, is necessary for the development of $\mathrm{K}^{+}$channel targeted drugs for glioma.

\section{Acknowledgement}

This project was supported by the National Natural Science Foundation of China (No. 30970353), and the Science and Technology Plan Projects in Liaoning Province, China (No. 2015020568).

\section{References}

1. Pardo LA, Stuhmer W (2014) The roles of $\mathrm{K}(+)$ channels in cancer. Nat Rev Cancer14(1): 39-48.

2. Zhorov BS (2011) Interactions of drugs and toxins with permeant ions in potassium, sodium, and calcium channels. Ross Fiziol Zh Im I M Sechenova 97(7): 661-677.

3. Sato M, Kishida D, Miyazaki D (2019) A Patient with Limbic Encephalitis Associated with Anti-leucine-rich Glioma-inactivated 1 (LGI1) Antibody Presenting with Slowly Progressive Cognitive Impairment and Fluctuating Striatal Lesions. Intern Med 58(2): 287-291.

4. Wawrzkiewicz-Jałowiecka A, Trybek P, Machura $Ł$ (2018) Mechanosensitivity of the BK Channels in Human Glioblastoma Cells: Kinetics and Dynamical Complexity. J Membr Biol 251(5-6): 667-679.

5. Bielanska J, Hernandez-Losa J, Moline T (2012) Increased voltagedependent K+ channel Kv1.3 and Kv1.5 expression correlates with leiomyosarcoma aggressiveness. Oncol Lett 4(2): 227-230.
6. Jang SH, Choi SY, Ryu PD (2011) Anti-proliferative effect of Kv1.3 blockers in A549 human lung adenocarcinoma in vitro and in vivo. Eur J Pharmacol 651(1-3): 26-32.

7. Tian C, Zhu R, Zhu L (2014) Potassium channels: structures, diseases, and modulators. Chem Biol Drug Des 83(1): 1-26.

8. Huang X, Jan LY (2014) Targeting potassium channels in cancer. J Cell Biol 206(2): 151-162.

9. Lastraioli E, Lottini T, Bencini L (2015) hERG1 Potassium Channels: Novel Biomarkers in Human Solid Cancers. Biomed Res Int 896432:1-9.

10. Cázares-Ordonez V, Pardo LA (2017) Kv10. 1 potassium channel: from the brain to the tumors. Biochemistry and Cell Biology 95(5): 531-536.

11. Becchetti A (2011) Ion channels and transporters in cancer. 1. Ion channels and cell proliferation in cancer. Am J Physiol Cell Physiol 301(2): C255-C265.

12. Ryland KE, Svoboda LK, Vesely ED (2015) Polycomb dependent repression of the potassium channel encoding gene KCNA5 promotes cancer cell survival under conditions of stress. Oncogene 34(35): 45914600 .

13. Abdullaev IF, Rudkouskaya A, Mongin AA (2010) Calcium activated potassium channels BK and IK1 are functionally expressed in human gliomas but do not regulate cell proliferation. PLoS One 5(8): e12304-e12316.

14. Zuniga L, Zuniga R (2016) Understanding the Cap Structure in K2P Channels. Front Physiol 7: 228-232.

15. Huang L, Li B, Li W (2009) ATP-sensitive potassium channels control glioma cells proliferation by regulating ERK activity. Carcinogenesis 30(5): 737-744

16. Bai Y, Liao H, Liu T (2013) MiR-296-3p regulates cell growth and multidrug resistance of human glioblastoma by targeting ether-a-go-go (EAG1). Eur J Cancer 49(3): 710-724.

17. Wang J, Li Y, Jiang C (2015) MiR-133b contributes to arsenic induced apoptosis in U251 glioma cells by targeting the hERG channel. J Mol Neurosci 55(4): 985-994.

18. Weaver AK, Bomben VC, Sontheimer H (2006) Expression and function of calcium-activated potassium channels in human glioma cells. Glia 54(3): 223-233.

19. Rosa P, Sforna L, Carlomagno S (2017) Overexpression of Large Conductance Calcium Activated Potassium Channels in Human Glioblastoma Stem Like Cells and Their Role in Cell Migration. J Cell Physiol 232(9): 2478-2488.

20. Hoa NT, Ge L, Martini F (2016) Temozolomide induces the expression of the glioma Big Potassium (gBK) ion channel, while inhibiting fascin-1 expression: possible targets for glioma therapy. Expert Opin Ther Targets 20(10): 1155-1167.

21. Stegen B, Butz L, Klumpp L (2015) $\mathrm{Ca}^{2+}$-Activated IK K+ Channel Blockade Radiosensitizes Glioblastoma Cells. Mol Cancer Res 13(9): 1283-1295.

22. D’Alessandro G, Grimaldi A, Chece G (2016) KCa3.1 channel inhibition sensitizes malignant gliomas to temozolomide treatment. Oncotarget 7(21): 30781-30796.

23. Kinboshi M, Shimizu S, Mashimo T (2019) Down-regulation of astrocytic Kir4.1 channels during the audiogenic epileptogenesis in liucine-rich glioma-inactivated 1 (Lgi1) mutant rats. Int J Mol Sci 20(5)

24. Felipe A, Bielanska J, Comes N (2012) Targeting the voltage-dependent $\mathrm{K}(+)$ channels Kv1.3 and Kv1.5 as tumor biomarkers for cancer detection and prevention. Curr Med Chem 19(5): 661-674.

25. Sharifi N, Salmaninejad A, Ferdosi S (2016) HER2 gene amplification in patients with prostate cancer: Evaluating a CISH-based method. Oncol Lett 12(6): 4651-4658.

26. Qi M, Yang X, Zhang F (2014) ERG rearrangement is associated with prostate cancer-related death in Chinese prostate cancer patients. PLoS One 9(2): e84959-e84970. 
27. Arvind S, Arivazhagan A, Santosh V (2012) Differential expression of novel voltage gated potassium channel Kv 1.5 in astrocytomas and its impact on prognosis in glioblastoma. Br J Neurosurg 26(1): 16-20.

28. Debska-Vielhaber G, Godlewski MM, Kicinska A (2009) Largeconductance $\mathrm{K}+$ channel openers induce death of human glioma cells. J Physiol Pharmacol 60(4): 27-36.

29. Turner KL, Honasoge A, Robert SM (2014) A proinvasive role for the $\mathrm{Ca}(2+)$-activated $\mathrm{K}(+)$ channel $\mathrm{KCa} 3.1$ in malignant glioma. Glia 62(6): 971-981.

30. Ru Q, Tian X, Wu YX (2014) Voltage-gated and ATP-sensitive K+ channels are associated with cell proliferation and tumorigenesis of human glioma. Oncol Rep 31(2): 842-848.

31. Staudacher I, Jehle J, Staudacher K (2014) hERG K+ channel-dependent apoptosis and cell cycle arrest in human glioblastoma cells. PLoS One 9(2): e88164-e88174.

32. Preussat K, Beetz C, Schrey M (2003) Expression of voltage-gated potassium channels Kv1.3 and Kv1.5 in human gliomas. Neurosci Lett 346(1-2): 33-36.

33. Jehle J, Schweizer PA, Katus HA (2011) Novel roles for hERG K(+) channels in cell proliferation and apoptosis. Cell Death Dis 2: e193-e201.

34. Sales TT, Resende FF, Chaves NL (2016) Suppression of the Eag1 potassium channel sensitizes glioblastoma cells to injury caused by temozolomide. Oncol Lett 12(4): 2581-2589.

35. Patt S, Preussat K, Beetz C (2004) Expression of ether a go-go potassium channels in human gliomas. Neurosci Lett 368(3): 249-253.

36. Catacuzzeno L, Aiello F, Fioretti B (2011) Serum-activated K and Cl currents underlay U87-MG glioblastoma cell migration. J Cell Physiol 226(7): 1926-1933.

37. Zhu J, Yan J, Thornhill WB (2014) The Kv1.3 potassium channel is localized to the cis-Golgi and Kv1.6 is localized to the endoplasmic reticulum in rat astrocytes. FEBS J 281(15): 3433-3445.

38. Venturini E, Leanza L, Azzolini M (2017) Targeting the potassium channel Kv1.3 kills glioblastoma cells. Neurosignals 25(1): 26-38.

39. Macfarlane SN, Sontheimer H (2000) Changes in ion channel expression accompany cell cycle progression of spinal cord astrocytes. Glia 30(1): 39-48.

40. Klumpp L, Sezgin EC, Skardelly M (2018) KCa3. 1 channels and glioblastoma: in vitro studies. Current neuropharmacology 16(5): 627 635.

41. Shah NH, Aizenman E (2014) Voltage-gated potassium channels at the crossroads of neuronal function, ischemic tolerance, and neurodegeneration. Transl Stroke Res 5(1): 38-58.

42. Hu L, Li LL, Lin ZG (2014) Blockage of potassium channel inhibits proliferation of glioma cells via increasing reactive oxygen species. Oncol Res 22(1): 57-65.

43. Ru Q Tian X, Pi MS (2015) Voltage gated K+ channel blocker quinidine inhibits proliferation and induces apoptosis by regulating expression of microRNAs in human glioma U87MG cells. Int J Oncol 46(2): 833-840.

44. Weiger TM, Colombatto S, Kainz V (2007) Potassium channel blockers quinidine and caesium halt cell proliferation in C6 glioma cells via a polyamine dependent mechanism. Biochem Soc Trans 35(Pt 2): 391395.
45. Hao X, Li X (2015) The knockdown of TASK-1 channels improved the proliferation of N2A cells. J Mol Neurosci 55(2): 314-317.

46. Bednarczyk P, Kowalczyk JE, Beresewicz M (2010) Identification of a voltage-gated potassium channel in gerbil hippocampal mitochondria. Biochem Biophys Res Commun 397(3): 614-620.

47. Song MS, Ryu PD, Lee SY (2017) Kv3.4 is modulated by HIF-1alpha to protect SH-SY5Y cells against oxidative stress-induced neural cell death. Sci Rep 7(1): 2075-2090.

48. Szabo I, Bock J, Grassme H (2008) Mitochondrial potassium channel Kv1.3 mediates Bax-induced apoptosis in lymphocytes. Proc Natl Acad Sci U S A 105(39): 14861-14866.

49. Cheng Y, Gulbins E, Siemen D (2011) Activation of the permeability transition pore by Bax via inhibition of the mitochondrial BK channel. Cell Physiol Biochem 27(3-4): 191-200.

50. Ru Q Li W, Xiong Q (2018) Voltage-gated potassium channel blocker 4-aminopyridine induces glioma cell apoptosis by reducing expression of microRNA-10b-5p. Molecular biology of the cell 29(9): 1125-1136.

51. Li X, Hu X, Li X (2015) Overexpression of tau downregulated the mRNA levels of Kv channels and improved proliferation in N2A cells. PLoS One 10(1): e116628-e116643.

52. Shen Z, Yang Q You Q (2009) Researches toward potassium channels on tumor progressions. Curr Top Med Chem 9(4): 322-329.

53. Yang KB, Zhao SG, Liu YH (2009) Tetraethylammonium inhibits glioma cells via increasing production of intracellular reactive oxygen species. Chemotherapy 55(5): 372-380.

54. Brown BM, Pressley B, Wulff H (2018) KCa3.1 Channel Modulators as Potential Therapeutic Compounds for Glioblastoma. Current neuropharmacology 16(5): 618-626

55. D'Alessandro G, Catalano M, Sciaccaluga M (2013) KCa3.1 channels are involved in the infiltrative behavior of glioblastoma in vivo. Cell Death Dis 4: 30781-30796.

56. D’Alessandro G, Grimaldi A, Chece G (2016) KCa3.1 channel inhibition sensitizes malignant gliomas to temozo- lomide treatment. Oncotarget 7(21): 30781-30796

57. Ge L, Hoa NT, Wilson Z (2014) Big Potassium (BK) ion channels in biology, disease and possible targets for cancer immunotherapy. Int Immunopharmacol 22(2): 427-443

58. Huang MH, Huang YM, Wu SN (2015) The Inhibition by Oxaliplatin, a Platinum-Based Anti-Neoplastic Agent, of the Activity of IntermediateConductance $\mathrm{Ca}^{2+}$-Activated $\mathrm{K}^{+}$Channels in Human Glioma Cells. Cell Physiol Biochem 37(4): 1390-1406

59. Thuringer D, Chanteloup G, Boucher J (2017) Modulation of the inwardly rectifying potassium channel Kir4.1 by the pro-invasive miR-5096 in glioblastoma cells. Oncotarget 8(23): 37681-37693.

60. Veeravalli KK, Ponnala S, Chetty C (2012) Integrin $\alpha 9 \beta 1$-mediated cell migration in glioblastoma via SSAT and Kir4.2 potassium channel pathway. Cell Signal 24(1): 272-281.

61. Huang L, Li B, Tang S (2015) Mitochondrial KATP Channels Control Glioma Radioresistance by Regulating ROS-Induced ERK Activation. Mol Neurobiol 52(1): 626-637. 
ISSN: 2574-1241

DOI: 10.26717/BJSTR.2019.16.002879

Wei Zou. Biomed J Sci \& Tech Res

(c) (P) This work is licensed under Creative

Submission Link: https://biomedres.us/submit-manuscript.php

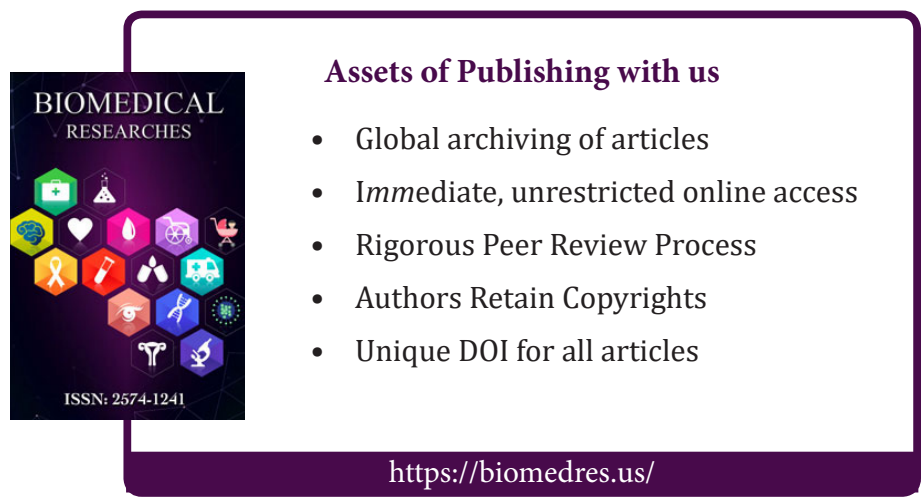

